\title{
Les programmes de géographie du lycée en France de 1995 à 2019 : un cas d'instabilité disciplinaire
}

Les programmes de géographie des lycées parus en 2019 n'ont suscité que peu de réactions chez les géographes ${ }^{1}$ et aucun débat dans la société française, à la différence une nouvelle fois des programmes d'histoire, plus sensibles au « poids du politique » (De Cock, 2017).

Ils n'ont pas fait l'objet d'une analyse sur la période récente, les auteurs se saisissant de la discipline en son entier, à propos d'un thème d'étude : l'environnement et le développement durable (Vergnolle-Mainar, 2011; Leininger-Frézal, 2009), l'aménagement (Leininger-Frézal et Carré, 2016) et/ou en se focalisant sur des traductions possibles de programmes (Gaujal, 2016; Robic et Rosemberg, 2016 ; Thémines, 2011).

Des études récentes se sont attachées à la fabrique des programmes, dévoilant des jeux complexes d'acteurs (D'Enfert, Legris et Verneuil, 2019). Intervenant après de longs « circuits d'écriture » (Legris, 2010), l'officialisation du programme, par arrêté ministériel, en fait un élément central des prescriptions d'enseignement. Ces dernières, qui sont avec les recommandations, les pratiques et les représentations de I'enseignement, un des quatre « espaces d'actualisation » de toute discipline scolaire (Reuter, 2008) ${ }^{2}$, portent et condensent dès lors une norme institutionnelle, pour nous celle de l'enseignement de la géographie en lycée.

La période 1995-2019 a pour cohérence a priori de rassembler quatre générations de programmes marquées par une ouverture relative de leurs circuits d'écriture (Legris, 2010), la massification des lycées ${ }^{3}$ et l'introduction d'un nouveau référentiel de l'action publique dans I'Education nationale (Dutercq et Maroy, 2013 ; Mons, 2007).

L'hypothèse qui fonde notre analyse est que la norme institutionnelle portée par les programmes ne concerne pas seulement les contenus enseignés. Elle lie ces derniers avec une définition du travail scolaire (des professeurs et des élèves), une façon d'entrer en rapport avec le groupe professionnel enseignant et un projet de socialisation politique en direction des élèves. Notre question est de savoir quels impératifs politiques gouvernent l'écriture des programmes du lycée en géographie, quels usages y sont faits des références scientifiques, à quelles valeurs orientant la définition du travail scolaire et le projet de socialisation des jeunes, les contenus de géographie prescrits se trouvent ainsi associés.

L'analyse textuelle menée emprunte à l'analyse de discours en privilégiant leur dimension énonciative. Elle met en évidence une succession de normes différentes, de formes diverses d'usage de la référence scientifique et de projets de socialisation aux orientations parfois antagoniques.

\footnotetext{
${ }^{1}$ Hormis, à notre connaissance, quelques géographes également didacticiens de la géographie (Genevois et Frézal-Leininger, 2019).

${ }^{2}$ La notion d' « espace d'actualisation » s'inscrit dans une approche non holiste des disciplines scolaires en didactiques. On considère qu'une discipline scolaire est une construction sociale qui prend des formes diverses selon les degrés, les filières, les périodes historiques, mais aussi selon les acteurs de cette construction, par exemple l'organisation, qui agit plutôt dans le secteur de la prescription et des recommandations, le corps social dans celui de la prescription (groupes de pression) comme celui les pratiques (demande envers les enseignants).

${ }^{3}$ Cette massification est pour une grande partie la conséquence de la création des filières de baccalauréat professionnel. Nous analyserons cependant les seuls programmes de lycée général; ceux des lycées professionnels nécessitant une contextualisation aux enjeux encore différents.
} 


\section{Les programmes de géographie de 1995 à 2019 : contextes de production et d'analyse d'une norme institutionnelle}

Relativement à la géographie scientifique, la géographie scolaire est dotée d'une grande autonomie de fonctionnement (Lefort, 2010 ; Chevalier, 1997) qui résulte de sa forte dépendance aux contraintes politiques, idéologiques et sociales du champ scolaire.

\section{Les programmes de géographie du lycée et les politiques scolaires (1995-2019)}

L'histoire des programmes de géographie, qui sont toujours en France ceux de «I'histoire et géographie » (1995, 2002, 2010) ou de «I'histoire-géographie » (2019) montre la prégnance des enjeux politiques : ce «lestage idéologique [...] consubstantiel à son installation et à son évolution : fabrication du socle républicain (1870-1940), affirmation d'une lecture du monde selon la trame des puissances (programme de terminale en 1905), alourdissement des horaires d'une géographie nationalo-centrée sous Vichy (Lefort, 1992), construction européenne et reformatage des échelles régionales (à partir des années 1980) jusqu'à la fin annoncée de la géographie (et de l'histoire) dans les programmes des classes terminales scientifiques, sociologiquement les plus valorisées (2009) 》 (Lefort, 2010). Le tableau $n^{\circ} 1$ en montre les derniers développements avec, depuis les années 2000, l'entrée du développement durable et de la mondialisation économique (tableau $\mathrm{n}^{\circ} 1$ ).

L'histoire des programmes s'imbrique dans une histoire des politiques scolaires. La période 1995-2020 est caractérisée par : $1^{\circ}$ une ouverture relative des circuits d'écriture des programmes; $2^{\circ}$ une massification de l'accès aux études en lycée ${ }^{4} ; 3^{\circ}$ l'adoption $^{\prime}$ des cadres de référence européens d'organisation du service public d'éducation (competence-based curriculum ${ }^{5}$, référentiels métier ${ }^{6}$ pour les personnels, évaluation de performance $\left.{ }^{7}\right) ; 4^{\circ}$ un rythme des réformes qui se cale sur l'agenda politique des majorités présidentielles.

Les programmes parus en $1995^{8}$ sont les premiers à être élaborés sous l'égide du Conseil National des Programmes (CNP), créé en 1990 pour mettre en œuvre une réforme des contenus pensée en fonction de principes communs et transversalement aux disciplines. Au CNP, succèdent en 2005 le Haut Conseil de I'Ecole, puis en 2013-2014 le Conseil Supérieur des Programmes (CSP), toutes instances émettant des avis et formulant des propositions aux ministères. L'élaboration des programmes est un terrain de tensions entre les Groupes Techniques Disciplinaires, puis Groupes d'experts, I'Inspection Générale de I'Education Nationale (IGEN) et les représentants des enseignants, syndicats et associations de spécialistes. La composition de ces instances, la nomination de leurs

\footnotetext{
${ }^{4}$ La proportion de bacheliers dans une génération est de $25,4 \%$ en $1980,29,4 \%$ en $1985,43,5 \%$ en 1990 , $62,7 \%$ en $1995,62,8 \%$ en $2000,61,2 \%$ en $2005,65 \%$ en 2010 et $78,6 \%$ en 2016 (Etat de l'enseignement supérieur et de la recherche en France, 2017, p. 14)

${ }^{5}$ L'expression désigne les programmes qui mettent l'accent sur les résultats de processus d'apprentissage : des connaissances, des compétences et des attitudes que les élèves ou étudiants doivent maîtriser plutôt que sur une définition des contenus à apprendre (content based curriculum). Cette démarche est soutenue par le Parlement européen et le Conseil de I'Union européenne qui ont adopté le 18 décembre 2006 une recommandation sur les compétences clés pour l'éducation et la formation tout au long de la vie (Journal officiel de I'Union Européenne, 394 du 30 décembre 2006).

${ }^{6}$ Un référentiel professionnel a pour fonction de décrire les attentes d'une organisation ou d'une collectivité à l'égard d'un professionnel dans l'exercice de son travail. Sa généralisation répond à des visées de pilotage du changement dans les professions comme de formation et d'évaluation des personnels. Leur formulation en compétences peut se révéler tout à fait insuffisante au regard de l'engagement des personnes nécessaire à leur activité.

${ }^{7}$ L'évaluation de performance est un mode de gestion des organisations scolaires et du travail des acteurs par les résultats, au nom d'une amélioration de l'efficacité et de la qualité du service d'éducation. Elle se traduit entre autres par la mise en œuvre d'enquêtes internationales sur les acquis des élèves.

${ }^{8}$ Ils ont été élaborés en histoire-géographie par le GTD Bernstein-Borne, auquel participent les géographes Robert Ferras et Philippe Pinchemel.
} 
présidents tout comme les délais que leur imposent les ministères sont susceptibles d'aviver les tensions'

De plus, les programmes de lycée de 1995 sont ceux de la Rénovation pédagogique des lycées qui, à partir de 1992, cherche à répondre à I'afflux massif d'élèves que produit la réorganisation des filières conduisant au baccalauréat. Elle met en œuvre I'objectif assigné au système éducatif depuis 1985 et officialisé dans la Loi d'orientation sur l'éducation de 1989 (gouvernement Rocard, ministre Jospin) de conduire « d'ici dix ans l'ensemble d'une classe d'âge au niveau du CAP ou du BEP et $80 \%$ au niveau du baccalauréat » (article 3). Cet objectif est confirmé par la Loi d'orientation et de programmation de 2005 (gouvernement Juppé). Les programmes de 2019 sont ceux de la dernière réforme en date du lycée (gouvernement Philippe, ministre Blanquer), qui met l'accent sur la mise en place d'un continuum lycée-Université.

S'agissant de curriculum ${ }^{10}$, la loi de 2005 instaure, en amont du lycée, un Socle commun de connaissances et de compétences (Article 9 de la loi du 23 avril 2005). Le CSP installé par la loi du 8 juillet 2013 d'orientation et de programmation pour la refondation de l'école (gouvernement Ayraud, ministre Peillon) met en place le deuxième Socle de connaissances, de compétences et de culture (Bulletin officiel $n^{\circ} 17$ du 23 avril 2015). Celui-ci marque un tournant dans l'écriture de contenus, organisée par compétences transversales (competence-based curriculum) plutôt que par périmètres disciplinaires

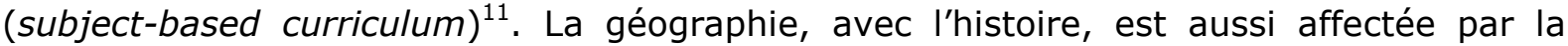
réforme du lycée amorcée en 2008 par le ministre Darcos (gouvernement Fillon) et terminée par la mission Descoings, avec la suppression de leur enseignement de la classe de terminale de la voie scientifique ${ }^{12}$. La réforme de 2019 met fin aux séries au profit d'une distinction entre enseignements communs et de spécialités. L'histoire-géographie fait partie du tronc commun évalué en contrôle continu dès la classe de première. L'histoire géographie géopolitique et sciences politiques apparaît comme nouvel enseignement de spécialité en première et terminale.

Enfin, concernant les professeurs, la loi de 2005 a introduit le premier référentiel de « compétences professionnelles des maîtres» (BOEN, $n^{\circ} 1,4$ janvier 2007), auquel succède un deuxième référentiel de «compétences professionnelles des métiers du professorat et de l'éducation » annexé à la loi de 2013 (BOEN, 23 juillet 2013).

\section{Le programme comme norme institutionnelle : images de la référence, du professeur et de l'élève}

Tout programme scolaire en géographie s'inscrit donc dans un ensemble de contraintes spécifiques du champ scolaire (politiques, curriculaires, gestionnaires). Mais il entretient aussi des rapports avec le champ scientifique. Ces rapports s'établissent par les personnes qui interviennent dans le processus d'écriture, dont certaines ont un statut double (universitaire et membre de I'IGEN), par les thèmes ou les concepts scientifiques

\footnotetext{
9 Les travaux du CSP sur les programmes parus en 2019 ont été marqués par des débats internes sur l'indépendance de l'instance, après la démission de son président le géographe Michel Lussault et de sa viceprésidente la linguiste Sylvie Plane, issus du quinquennat Hollande et la nomination par le ministre Blanquer d'une nouvelle présidente, la philosophe Souâd Ayada.

${ }^{10}$ Issue du monde anglo-saxon, la notion de curriculum est plus large que celle de programme scolaire en France. Le curriculum désigne une conception d'ensemble, structurée et cohérente, de directives selon lesquelles organiser les apprentissages scolaires en fonction de résultats visés.

${ }^{11}$ La présentation de ces compétences en cinq domaines (Les langages pour penser et communiquer, les méthodes et outils pour apprendre, la formation de la personne et du citoyen, les systèmes naturels et les systèmes techniques, les représentations du monde et l'activité humaine) précède celle des enseignements qui permettent de les construire. La notion de discipline disparaît de ces textes au profit de celle d'enseignement.

12 L'écriture de nouveaux programmes aboutit en 2010. Elle est confiée en histoire-géographie à un groupe d'experts co-présidé par Michel Hagnerelle, groupe auquel appartient la géographe Nacima Baron Yellès.
} 
qui peuvent être mobilisés ou encore par une relation plus diffuse et implicite que le programme installe avec la référence scientifique en général.

Parce qu'il s'adresse à des enseignants, tout programme correspond enfin à ce qui, en analyse du travail, est appelé travail prescrit (Davezies, 1993) : ce qu'une organisation définit comme la tâche à réaliser par ses agents, ce qu'ils ont à faire.

On doit donc considérer que le programme délivre une norme institutionnelle ${ }^{13}$ en matière non seulement de contenus, mais aussi de production de savoir scolaire, c'est-àdire de pratiques professorales et d'attentes éducatives à leur endroit. Comme toute norme, elle élit « une manière de faire qui répond à des attentes et/ou à des valeurs, parmi un ensemble ouvert de possibles »(Prairat, 2015, p. 7). Pour avancer dans I'identification de cette norme, nous mettrons en œuvre des notions que nous proposons, d'images de la référence, du professeur et de l'élève.

L'image de la référence concerne la justification du choix de contenus du programme. L'expression renvoie à une problématique centrale dans le champ des didactiques ${ }^{14}$ qui est celle de la référence. Cette problématique met l'accent sur le « sens politique et en tout cas social » (Martinand, 2001, p. 19) de tout choix de contenus. L'idée d'une image de la référence permet de se saisir du programme, comme d'un acte de justification de contenus qu'il met en forme en les articulant à un projet politique. L'image du professeur est I'enseignant attendu pour la réalisation disciplinaire de ce projet politique, sous le double aspect des compétences qui lui sont reconnues et des rapports qu'il doit installer chez les élèves avec la géographie enseignée. L'image de l'élève est la personne à former que le programme invoque, en formulant les buts de l'enseignement et en décrivant la nature des apprentissages visés et du travail attendu du professeur.

Sans s'inscrire pleinement dans le domaine de pertinence de la sociologie de la critique ${ }^{15}$ (Boltanski et Thévenot, 1991, Derouet, 2000), les images de la référence, de l'enseignant et de l'élève permettent cependant de circonscrire un principe supérieur, une logique de justification qui fonde la hiérarchie des valeurs attribuées aux contenus choisis, au travail enseignant attendu et aux apprentissages visés. Ce principe n'est pas explicitement formulé dans un programme, mais il peut être inféré d'indices textuels que I'analyse va mettre au jour et organiser.

\section{Mise en œuvre méthodologique de l'analyse de discours}

L'analyse menée sur les programmes est une analyse de discours qui s'est attachée à l'énonciation, c'est-à-dire à l'utilisation de la langue dans ce contexte précis de communication. Une simple mise en série de ces textes fait apparaître qu'ils ne sont que très partiellement des listes de contenus et que, sous I'appellation générique de programmes, ils abritent des constructions discursives différentes. L'analyse de l'énonciation s'est concentrée sur les procédés d'effacement énonciatif par lesquels, dans certains genres de discours auxquels appartiennent les textes officiels, le locuteur donne l'impression qu'il se retire de l'énonciation (Vion, 2001). Pour les linguistes (KerbratOrecchioni, 1980; Philippe, 2002), Or «l'effacement énonciatif est un simulacre

\footnotetext{
13 «Une norme est une régularité qui enferme une injonction à faire ou à ne pas faire, elle contraint au sens étymologique du terme (constringere signifie en latin serrer), elle resserre le champ des possibles. Elle a enfin une dimension collective [...] elle concerne toujours une pluralité humaine » (Prairat, 2015, p. 7). La norme institutionnelle est définie par l'organisation, alors que la norme professionnelle est propre au groupe professionnel qu'elle emploie ; ce dernier opérant souvent par «renormalisations » à partir de normes antécédentes (Lantheaume et Simonian, 2012).

${ }_{14}$ Les didactiques sont entendues non comme des pratiques ou des prescriptions d'enseignement, mais comme les disciplines scientifiques qui étudient les contenus en tant qu'ils sont objets d'enseignement et d'apprentissage (Reuter, 2008).

${ }^{15}$ Même si notre analyse ne s'attache pas à des justifications, des argumentations en situation d'interaction, de co-présence, notre matériau textuel présente une dimension argumentative.
} 
linguistique, relevant d'une stratégie énonciative $[\ldots] ;[.$.$] propre à certains genres de$ discours dans lesquels le locuteur a intérêt à afficher un ethos de neutralité et d'objectivité $^{16}$; cet ethos apparent [dissimulant] souvent des effets argumentatifs plus complexes, dont les enjeux sont interactionnels, interprétatifs, voire éthiques 》 (Provenzano, 2010). Autrement dit, alors que le genre textuel le porte à l'effacement, le locuteur exprime des choix stratégiques dans ce contexte de communication. La question est donc de savoir quels enjeux d'enseignement disciplinaire sont construits à travers des prises de position neutralisées dans l'acte de langage même.

\section{La géographie prescrite du lycée : changement de programme, changement de norme institutionnelle}

\section{Effacement énonciatif et variations des objets de discours}

L'analyse des procédés d'effacement énonciatif a été conduite en prenant en compte le plan discursif, avec la construction d'objets ${ }^{17}$; le plan rhétorique et stylistique avec les procédés de dynamisation des objets construits $^{18}$; et le plan linguistique avec les énoncés « objectivants » à caractère générique, doxique et axiologique ${ }^{19}$ (Rabatel, 2004). Nous présentons les résultats de cette analyse en montrant les variations d'un texte à l'autre. Le tableau $n^{\circ} 2$ propose une vue synoptique de ces résultats, en les organisant par rapport aux trois catégories d'analyse proposées, à savoir l'image de la référence, l'image du professeur et l'image de l'élève.

\section{5 : I'enseignement comme projet commun}

Dans le programme de 1995, I'objet élaboré sur le plan discursif (voir tableau n²) est «l'enseignement de l'histoire et de la géographie». Cet objet est doté d'un «projet éducatif » et caractérisé au moyen d'énoncés axiologiques : «l'enseignement doit être problématique $»^{20}$ ou génériques : «Le document dans ses divers états est constitutif de l'enseignement de l'histoire et de la géographie $»^{21}$. De ce premier objet, est dérivé l'objet «programmes», lequel est établi par des énoncés axiologiques : «Les programmes d'histoire et de géographie du lycée [...] doivent enfin, et c'est l'essentiel, répondre à des finalités (connaissances, apprentissage de la réflexion, exercice de la raison critique) 》; «Les programmes, tant en histoire qu'en géographie, sont construits pour induire une démarche plus synthétique qu'analytique. Au lycée l'enseignement doit être problématique : la recherche du sens est primordiale [...]». Le texte officiel se conclut sur une liste commentée de contenus. Cette liste est complétée pour la classe de seconde d'une courte introduction qui disparaît quasiment en classe de première et de terminale, où la désignation du contenu se suffit à elle-même.

\footnotetext{
${ }^{16}$ Les genres de discours associés à ce type de stratégie sont scientifiques, publicitaires, journalistiques ou juridiques (Vion, 2001).

17 La construction de l'objet est conditionnée à l'effacement apparent de l'énonciateur. L'analyse vise

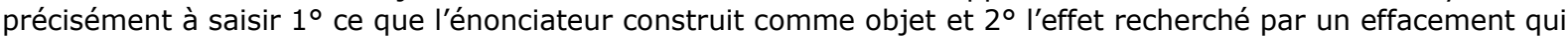
construit cet objet plutôt qu'un autre.

${ }^{18}$ Procédés permettant de considérer que les objets construits existent d'eux-mêmes, sont dotés d'une efficace propre. Par exemple : la géographie montre aux élèves que ; les programmes d'histoire-géographie donnent une vision.

${ }_{19}$ Les énoncés génériques établissent des propositions à caractère très général, qui peuvent passer pour universellement vraies, voire confiner à la maxime. Les énoncés doxiques supposent un savoir partagé ou à faire partager par une communauté. Les énoncés axiologiques correspondent à un jugement sur la valeur morale (bien/mal, juste/injuste) d'une proposition. Ce jugement attribue une vertu, un mérite (ou leur contraire) à une personne, une situation, un comportement, etc.

${ }^{20} \mathrm{Ce}$ qui équivaut à dire qu'il est bien ou juste que « l'enseignement » soit «problématique » et qu'il est bien ou juste de penser qu'il doit en être ainsi.

${ }^{21}$ Cet énoncé se donne comme vérité, permettant ainsi de lier les disciplines savantes et l'histoire et la géographie au lycée.
} 
La stratégie énonciative consiste à s'effacer pour installer dès la première phrase un principe auquel la prescription se déclare subordonnée : «L'enseignement secondaire français associe l'histoire et la géographie, sciences sociales historiquement liées, et que leur finalité civique et éducative rapproche ». L'énoncé princeps est prolongé par des énoncés génériques sur les finalités ${ }^{22}$ et les méthodes : "Les méthodes mises en œuvre dans l'enseignement de l'histoire et de la géographie placent la dimension critique au centre des pratiques pédagogiques. Elles sont en elles-mêmes éducatives ».

Le rapport avec la profession est amené par une formule passive invoquant « une ancienne tradition » permettant de reconnaître le principe de liberté pédagogique. Le passage consacré aux «styles pédagogiques» débouche sur l'énoncé axiologique suivant : "Plus qu'un style pédagogique, c'est l'acquisition organisée des connaissances qui est essentielle ». La profession se voit ainsi fixée une norme de visée plus que de conduite, dans le cadre du «projet éducatif » que porte « l'enseignement de l'histoire et de la géographie».

\section{2 : les mots de la géographie}

Les textes officiels de 2000 puis 2002 construisent un objet « programmes » qu'ils installent par des énoncés doxiques «[ils] permettent en effet la compréhension du monde contemporain, par l'étude de moments historiques qui ont participé à sa construction et par celle de l'action actuelle des sociétés sur leurs territoires ». Pour la classe de seconde, les énoncés génériques ne sont pas développés pour des finalités ou des méthodes, mais pour des contenus de savoir. Le discours prend la forme d'un glossaire de «notions » articulées entre elles. Par exemple : «L'aménagement désigne à la fois un ensemble d'actions d'une société et le résultat de ces actions sur son territoire. L'aménagement est donc le fruit d'acteurs qui, dotés de leurs stratégies, de leurs représentations spatiales, sont producteurs d'espace $[. ..] \gg$. Les notions sont les objets du discours: «La notion d'organisation de l'espace est au cœur de l'ensemble du programme, abordée à travers deux entrées principales [...]». Une logique hypertextuelle (utilisant la couleur rouge pour distinguer les notions) permet de passer du commentaire général au commentaire par thème et à une liste différenciée de « notion centrale du programme » (organisation de l'espace), de «notions transversales du programme » (environnement, aménagement) et $\mathrm{d}^{\prime} \ll$ autres notions de base ». Dans les autres programmes, les énoncés doxiques à formules impersonnelles mettent en avant un contenu : «On montre que la France [...] » ou un procédé : «On présente d'abord les grands traits de [...] ». Ils rejoignent des caractéristiques d'énonciation prévalant pour les trois niveaux du programme de 1995.

Si le principe de la liberté pédagogique est rappelé au moyen d'énoncés génériques : «Les enseignants déterminent leurs approches pédagogiques » (seconde), des énoncés axiologiques accompagnent l'introduction des études de cas. "Leur choix est donc fondamental : elles doivent être représentatives et sont l'occasion de définir des enjeux, des problèmes à résoudre en analysant des situations géographiques diversifiées »; «II est nécessaire que chaque étude de cas soit contextualisée par une mise en perspective à plus petite échelle, s'appuyant prioritairement sur des cartes ». L'étude de cas accède ainsi au statut d'un objet doué d'autonomie : «Les études de cas [...] mettent en place les problématiques nécessaires à l'appropriation des savoirs et constituent l'apprentissage du raisonnement géographique ». Elles sont l'instrument qui permettra aux professeurs de donner accès, pour leurs élèves, aux savoirs définis par notions.

\footnotetext{
${ }^{22}$ Une finalité est un principe d'organisation et de justification du choix de savoirs à enseigner. Elle est partie intégrante d'une discipline scolaire : "la métamorphose d'un savoir en discipline incorpore ces finalités : elles guident par exemple le choix des exercices, de tel ou tel sujet... » (Prost, 1998).
} 


\section{0 : des objectifs d'apprentissage}

Comme en 2002, l'effacement énonciatif sert la construction d'un objet « programme ». Il substitue cependant des « objectifs d'apprentissage » aux finalités du programme. Une fois passée l'assertion selon laquelle « Le programme de géographie de la classe de seconde permet la [...] poursuite de l'étude de l'organisation, de l'aménagement et du développement des territoires à toutes les échelles », on ne trouve pas de glossaire de notions. De plus, des énoncés doxiques installent l'objet «développement durable ». La proposition : « Le développement durable apparaît ainsi comme une autre façon de lire le monde, de le penser et de le gérer », permet à cet objet de discours de prendre la place que «la géographie » avait dans le programme de 1995 : «la géographie [...] constitue une des clés d'explication du monde contemporain ».

La liberté s'accompagne désormais de la « responsabilité pédagogique » (programme de seconde) et doit prendre en compte la catégorie nouvelle des «objectifs d'apprentissage $\gg^{23}$. Ces derniers prennent la forme d'un tableau organisé en familles de « capacités et méthodes » valant pour I'histoire et la géographie. Les présentations de thèmes sont détaillées en tableaux comportant une colonne «questions » et une colonne « mise en œuvre». Cette dernière propose des listes d'objets, sans commentaire relatif à leur articulation ou leur traitement, en dehors d'une référence répétée à la démarche d'étude de cas. L'énoncé axiologique selon lequel «Dans la construction de son enseignement [...] le professeur vise comme objectifs d'apprentissage les connaissances, les capacités et les méthodes définies dans les programmes », redéfinit la nature de ce qui est désigné par enseignement. En 1995, l'enseignement est une production collective de laquelle participe l'énonciateur ; en 2010, il relève de la responsabilité individuelle de chaque professeur. Comme en 2002, les caractéristiques du programme de seconde ne se retrouvent pas nettement dans les deux autres niveaux. Dynamisant l'objet «programme», le discours y minimise la responsabilité du professeur : «Très rénové dans sa conception, son organisation et ses problématiques, il [le programme] privilégie les approches problématisées [...] il revêt une dimension éminemment citoyenne, invitant les élèves à prendre conscience de la dimension prospective et stratégique de tout choix d'aménagement » (classe de première).

\section{9 : place à la discipline}

Le discours des programmes de 2019 construit comme objet premier la « discipline». Une série d'énoncés doxiques mettant en avant «I'histoire et la géographie enseignées » précise ses propriétés : elles «transmettent aux élèves des connaissances [...]. Elles les aident à acquérir des repères temporels et spatiaux; elles leur permettent de discerner l'évolution des sociétés [...] ainsi que les actions et décisions des acteurs ; elles les confrontent à l'altérité [...] 》. De la même façon, «L'enseignement», selon une formule qui met à distance les enseignants, «développe des connaissances et construit des capacités $[\ldots]$ ».

Les finalités ne participent pas de cette construction discursive de la discipline. Très développés, les contenus proposent non un glossaire de notions, mais une description du monde. «Le monde est profondément transformé par les mobilités. Celles-ci peuvent être motivées par de nombreux facteurs (fuir un danger, vivre mieux, travailler, étudier, s'enrichir, visiter...) [...] ». Quelques énoncés doxiques indiquent une direction de travail : «Il s'agit de réfléchir aux enjeux liés au développement différencié de la population dans le monde, en questionnant la relation entre développement et inégalités ». Ces programmes gardent de ceux de 2010 un tableau de capacités et de méthodes. De trois catégories, ce tableau est passé à deux, au détriment de la catégorie «Maîtriser des

\footnotetext{
${ }^{23}$ Un objectif formule ce qu'un élève ou un étudiant est censé maîtriser à la fin d'un enseignement.
} 
méthodes de travail personnel ». Une liste de «notions et vocabulaire à maîtriser [...] » est proposée en fin de programme. A la différence de 2002, cette liste n'est pas reliée aux tableaux qui précédent.

La notion de liberté pédagogique est absente. Un énoncé doxique formule des attentes qui sont situées dans le domaine des conduites : « [...] l'enseignement associe des temps dédiés : - à la transmission des connaissances par les professeurs et d'écoute active de la part des élèves ; - à l'étude de sources, à l'analyse approfondie et critique de documents variés (cartes, textes, iconographie, vidéos...) et à la réalisation de croquis ».

\section{La pluralité successive des mondes de la géographie du lycée}

De ces analyses, il est difficile de dégager des évolutions sur la période. Entre 1995 et 2019, la tendance est à l'effacement des finalités au profit des contenus. Mais le programme de seconde de 2010 est une exception par la brièveté de ces derniers. Si les programmes de 1995 et ceux de 2019 sont d'une grande homogénéité formelle - et ce en raison de leur contribution ou concomitance avec des réformes du lycée - ceux de 2002 et de 2010 sont hétérogènes, associant la nouveauté en seconde à une certaine tradition en première et terminale. Ces analyses peuvent être condensées en une série de modèles spécifiques d'images de la référence, du professeur et de l'élève. Chaque génération de programme incarne ainsi une norme institutionnelle différente bien qu'inégalement aboutie.

\section{5 : un monde civique}

Les programmes de 1995 installent un monde civique où la référence est construite par assimilation de la formation intellectuelle ou critique et de l'éducation à la citoyenneté. C'est le motif originel de justification de l'enseignement de l'histoire et de la géographie, que ce programme ré-énonce de façon appuyée, presque solennelle. Le professeur attendu est construit comme l'associé d'un «projet éducatif de l'enseignement de I'histoire et de la géographie», capable de s'en saisir et de le servir avec son propre style, pourvu qu'il assure une «acquisition organisée des connaissances ». L'élève est institué comme un citoyen à doter d'une culture et d'une faculté de discernement nécessaires à la vie démocratique. Ce monde civique est caractérisé par l'antécédence de «l'enseignement » sur toute autre réalité, y compris celle du programme, la vigueur du discours sur les finalités, l'appel à la science comme ressource pour rénover I'enseignement. La question des pratiques enseignantes est réglée par l'invocation d'une démarche «synthétique » et «problématique » dont le respect garantit la réalisation du projet civique.

\section{2 : le monde de l'étude}

Le programme de la classe de seconde en 2002 construit un monde de l'étude où les savoirs sont fermement définis ainsi que l'étude de cas, démarche qui doit en organiser «l'appropriation». Symétriquement à cette expertise des savoirs placée du côté du «programme», le professeur attendu est reconnu dans son expertise spécifique de pédagogue, littéralement celui qui conduit les élèves aux savoirs. On pourrait dire que le programme se veut pédagogique pour les enseignants. L'élève à former est un apprenti que la pratique guidée de la démarche d'étude de cas assure d'une « compréhension dynamique et distanciée » du monde. Dans le monde de l'étude, l'insistance sur le magistère civique de la disciplinaire n'a guère de sens Les finalités et la citoyenneté sont 
conditionnées à une juste définition et mise en œuvre des contenus ${ }^{24}$. De même, le rapport à la science est mis en œuvre dans les définitions de contenu notionnel plus qu'il n'est affirmé comme principe antécédent.

\section{0 : le monde des référentiels}

Parmi les programmes de géographie 2010, celui de la classe de seconde est exemplaire du monde des référentiels de compétences qui s'installe dans l'Education nationale en 2005. Le cadrage du programme de géographie est double: des «objectifs d'apprentissage » méthodologiques d'une part et le développement durable «fil conducteur » de l'enseignement disciplinaire d'autre part. La figure du professeur est celle d'un enseignant autonome, responsable, capable de prendre en charge ces cadres de référence pour répondre aux attentes. Il sait combiner un fil conducteur non disciplinaire, une liste de capacités et de méthodes aux formulations assez générales ainsi que des thèmes d'étude disciplinaires dépourvus de commentaire. L'élève à former est comme en 2002 un apprenti à guider dans une «démarche géographique ». Mais cette démarche est désormais considérée comme «une contribution essentielle à l'éducation au développement durable »; elle est donc située en fonction d'un cadre autre que disciplinaire.

\section{9 : le monde de la tradition}

Les programmes de 2019 installent une norme apparentée au monde domestique (Boltanski et Thévenot, 1990) qui privilégie la référence à la tradition et à des formes classiques d'autorité. Le mot discipline avait disparu des programmes de seconde de 2002 et $2010^{25}$. Il est en 2019 l'objet principal construit par le discours. A l'inverse de l'enseignant responsable de 2010, la figure du professeur est décrite à partir d'une conduite attendue (un comportement). Le texte à dire est souvent amorcé dans le « commentaire » du programme. Il est reconnu au professeur la liberté de choisir les exemples pour développer ce texte. L'élève à former est un élève exercé : après «l'écoute active » du professeur, il avance chaque année dans «l'apprentissage des exercices de géographie » évalué en contrôle continu à partir de la classe de première.

\section{Usages de la référence scientifique et valeurs du travail scolaire: instabilité de l'organisation curriculaire}

Les mondes civique, de l'étude, des référentiels et de la tradition sont le produit d'une analyse de discours. Elle montre que les variations de la norme institutionnelle touchent tout autant l'organisation de contenus que le travail enseignant prescrit et les effets attendus chez les élèves. L'analyse se poursuit en précisant les lignes de force qui organisent chaque ensemble de programmes sur les trois années du lycée. Ces orientations rencontrent des limites ou comportent des formes d'inachèvement qui nous semblent confirmer l'état d'instabilité de la géographie scolaire prescrite.

\section{L'appel à la science et le volontarisme patrimonial}

\footnotetext{
${ }^{24}$ Ce qu'illustre le passage suivant : «Afin que ces finalités affichées soient réalisables, les contenus des programmes sont nettement délimités tout en préservant la liberté pédagogique des enseignants » (programme de seconde).

${ }^{25}$ En dehors de cet énoncé doxique qui a pour fonction d'en relativiser l'importance : « Toute étude du développement durable nécessite donc un croisement des regards, des savoirs et des méthodes des différentes disciplines » (programme de seconde, 2010).
} 
Dans les programmes de 1995, le rapport à la science est invoqué dans le moment initial de présentation du «projet éducatif» de «l'enseignement de l'histoire et de la géographie ». Le territoire est le vocable qui relie la géographie, reconnue avec I'histoire comme « sciences sociales constituées », au projet civique qu'elle doit servir : «L'État de droit est au service de la personne. Sa perfection est un objectif de la démocratie. Il doit être reconnu comme un patrimoine à connaître, à respecter et à enrichir. L'espace aménagé est placé sous la responsabilité du citoyen : il est à la fois héritage à préserver et territoire à transformer pour répondre aux besoins sociaux actuels ».

Ainsi utilisé, le mot territoire peut être rattaché à l'un des sens - celui d'espace approprié - que lui attribue Les mots de la géographie récemment paru (1992, p. 435-436). Mais il n'est pas l'objet d'un discours définitionnel. Aucun des mots du programme ne l'est ; ce en quoi il correspond à la position du co-président du GTD Dominique Borne. Celui-ci s'est opposé dans un débat public (Trema, 1994) à la possibilité d'un programme écrit en concepts, possibilité défendue par des géographes (Antoine Bailly, Roger Brunet, Gérard Hugonie $)^{26}$. Le territoire fonctionne comme un vocable performatif. Prescrire le territoire ainsi, c'est le faire en affirmant grâce à lui la force supérieure d'un projet éducatif qui est aussi un projet d'Europe ${ }^{27}$. La clé de voûte de ce programme est donc la classe de première : «En géographie, le programme s'ouvre sur une définition de l'Europe : l'approche de la France est donc renouvelée en fonction des réalités et des problématiques d'aujourd'hui [...] les élèves doivent saisir que le territoire national est une construction inscrite dans la durée et qu'il appartient à des ensembles divers. Il s'agit de comprendre comment la France est européenne par ses milieux, son histoire, ses cultures [...]». Une fois l'Europe installée en chaque lycéen comme le lieu d'où regarder le monde, le programme de terminale le donne à lire à tous, avec ses espaces de la puissance (I'Europe y est rattachée par l'Allemagne aux côtés du Japon et des Etats-Unis) et les continents ou quasi-continents où se posent les «problèmes du développement » (Afrique, Amérique du Sud, Russie, Chine, Inde).

Ce volontarisme patrimonial exclut du champ des emprunts l'apport d'une géographie environnementale renouvelée, en dépit de la proposition d'une «didactique de la géographie physique» (Bertrand, 1991) organisée par le «système conceptuel tripolaire: géosystème, territoire, paysage ». L'environnement n'est qu'un « thème » transversal (classe de seconde) et ses contenus sont formulés en « relations hommemilieu » et «milieux » européens. De la même façon, l'insistance mise sur « Les dimensions historique et culturelle [qui] prennent, à côté de l'économie, une place déterminante dans la réflexion » (programme de première) converge avec la mise à l'écart d'une géographie de la mondialisation pourtant accompagnée d'un important travail didactique (Retaillé, 1991), mais rejetée lors de son introduction en 1987 (Clerc, 2002).

La rhétorique de la référence à la science sert finalement l'assignation de la géographie scolaire à un projet de socialisation européenne des lycéens par « enracinement dans les territoires ${ }^{28}$ (Borne, 1994), valeur cardinale du travail demandé aux enseignants.

\footnotetext{
26 Dominique Borne fait valoir la culture géographique disciplinaire d'enseignants très majoritairement historiens que la nouveauté formelle d'un programme écrit en concepts dérouterait et conduirait à «un enseignement où se succèdent les définitions 》 (Borne, 1994). "Les niveaux d'enseignement sont d'abord définis par des espaces, qui entraînent ensuite des concepts, cela va de soi. Les espaces sont premiers. Et les concepts vieillissent 》 (ibid.).

27 Les ministres du moment, Jack Lang puis François Bayrou, sont attentifs à l'introduction de la dimension européenne dans les enseignements en contexte d'approfondissement de la construction européenne (Legris, 2010, p. 439).

28 « La finalité civique de la géographie c'est aussi d'enraciner la responsabilité et la liberté du citoyen, de l'enraciner dans les territoires, du local au régional, de la nation au monde », affirme Dominique Borne au Colloque de Montpellier de 1993 sur l'enseignement de la géographie.
} 


\section{Une médiation scientifique sous contraintes}

Avec son glossaire organisé en fonction $d^{\prime}$ « une même et unique problématique, celle de l'appropriation et de la gestion de l'espace par les sociétés » (programme de seconde), le programme de seconde de 2000 attache fortement les contenus prescrits à la géographie scientifique, L'écriture porte la marque du travail de ressaisie de leur discipline opéré par les géographes au tournant des années 1980-1990. Certains (Franck Auriac, Georges Bertrand, Armand Frémont, Yves Guermond, Philippe Pinchemel) avaient contribué par des propositions, aux débats ouverts par la Commission Gros-Bourdieu ${ }^{29}$ sur les «contenus de l'enseignement» (Brunet, 1989). C'est cette contribution et ses suites dont les apports n'ont pas été pris en compte par le GTD précédent.

Les deux niveaux de programme suivants sont élaborés dans un autre contexte : le ministre Lang succède au ministre Allègre et le GE présidé par le géographe Armand Frémont succède au GTD co-présidé par le géographe Jean-Paul Charvet. C'est toujours un objet «programme » qui est construit et il conserve en classe de première l'étude de cas, seule démarche d'apprentissage définie en 25 ans. Il s'agit d'«approfondir la démarche géographique engagée » à l'aide de «notions [sur lesquelles il s'agit de] faire réfléchir les élèves » (classe de première). La valeur qui gouverne les emprunts à la géographie scientifique est celle d'une formation aux problèmes de l'aménagement des espaces de vie, à la complexité des constructions et des interventions humaines.

Pourtant des difficultés sont apparues pour traduire en contenus la valeur, cardinale dans le monde de l'étude, d'un apprentissage de cette complexité. Une première difficulté est liée à l'urgence de devoir présenter un programme, après le refus essuyé par le premier projet : un programme unique associant histoire et géographie ${ }^{30}$. Le retour à une forme classique conduit à penser l'apport de formation civique en classe de première en partie en continuité avec le programme de 1995. Par exemple, la géographie de l'environnement en Europe (classe de première) est «naturalisée » au profit d'une Europe des milieux et de l'enseignement des politiques environnementales. Il est en rupture avec un programme de seconde qui déploie l'arsenal d'une géographie environnementale rénovée (notions d'environnement, géosystème, ressources, risques, etc. $)^{31}$.

Mais une seconde difficulté, plus fondamentale, concerne la conception même d'unee progression « dans la maîtrise des approches systémiques et dans la prise en compte de la complexité et de la hiérarchie des facteurs d'explication » affichée par le programme de seconde. Son écriture aurait nécessité d'articuler l'expression d'attentes graduées sur trois ans dans l'apprentissage de la pensée complexe en géographie, avec les contraintes posées par des logiques de programme variant chaque année : logique thématique en seconde, logique territoriale en première (I'Europe, la France) puis une combinaison des deux en terminale (les interdépendances constitutives de l'espace mondial et des espaces transnationaux : la façade atlantique de l'Amérique du Nord, I'Europe rhénane, l'espace méditerranéen).

\footnotetext{
${ }^{29}$ Créée à la demande du Ministre de l'Education nationale Lionel Jospin, pour ouvrir le chantier de la rénovation des contenus d'enseignement, cette commission co-présidée par le physicien François Gros et le sociologue Pierre Bourdieu est organisée en groupes disciplinaires. Dans le groupe présidé par I'historien Philippe Joutard pour l'enseignement de l'histoire, de la géographie et de l'éducation civique, figure le géographe Gérard Dorel, au titre de l'Association des Professeurs d'Histoire Géographie.

${ }^{30}$ Interviewé par Patricia Legris, Armand Frémont précise ce à quoi il avait alors pensé : « J'avais donné des exemples. Par exemple, "industrialisation et désindustrialisation en Europe au XXe siècle", à la fois en histoire et en géographie. Et pour prendre à l'autre bout, pensant amuser et troubler, "le football de sa fondation à maintenant" » (Legris, 2010, p. 485).

${ }^{31}$ La présence d'Yvette Veyret au CNP favorise I'installation de cette géographie.
} 
Si cette articulation n'est sans doute pas impossible logiquement, il faut bien reconnaître que l'abord de la complexité a été de fait fortement réduit. L'Europe est étudiée par « approches » de «la France en Europe », qui sont autant de principes d'organisation de l'espace (les Etats, les villes et leurs réseaux, les régions). Leur étude successive prévaut sur la recherche des interdépendances, contradictions, rétroactions, ajustements entre ces différents principes. Le programme de terminale réduit la difficulté en affirmant que les «études de cas et les exemples au choix disparaissent afin de répondre aux exigences du baccalauréat », ce qui ouvre la possibilité de descriptions faiblement problématisées.

Ces difficultés entraînent également un affaiblissement de la valeur socialisatrice du projet initié en classe de seconde. L'apprentissage de la complexité indique un horizon de réflexion politique sur «les effets sur les territoires des interventions des nombreux acteurs spatiaux, publics ou privés » (programme de seconde), quand le programme de terminale ajuste la valeur de la géographie enseignée à l'obtention de l'examen final ${ }^{32}$, lequel ne vise pas à faire la preuve d'une compréhension par les élèves de problèmes d'aménagement.

\section{Valeur et limites de l'enseignement de cadres pour l'action}

Le programme de 2010 articule de façon cohérente l'objet d'enseignement prescrit : les cadres de référence et enjeux de l'action publique en matière de gestion et d'aménagement des territoires, avec la manière de prescrire cet objet : les nouveaux codes de l'action publique en éducation (référentiels, appel à la responsabilisation de chaque enseignant). Ce faisant, la prescription opère un double déplacement: en attribuant au développement durable en classe de seconde, la fonction jusqu'alors accordée à la géographie de constituer une « grille de lecture » du monde ; en déplaçant I'horizon de travail de la discipline vers le monde de l'action et vers la prospective. Le programme met en question le cadre disciplinaire en choisissant de provoquer son ébranlement.

Cette orientation est favorisée par l'institutionnalisation du champ interdisciplinaire des sciences du territoire, même si la référence n'est pas explicite ${ }^{33}$. Elle résulte aussi d'un basculement de références en matière d'éducation environnementale, de I'Education Relative à I'Environnement (ERE) ${ }^{34}$ à l'Education au Développement Durable (EDD), dont Michel Hagnerelle, par ailleurs membre du GE, est I'un des acteurs en 2003-2004. Le rapport Bonhoure-Hagnerelle diagnostique l'éparpillement des initiatives et préconise une «stratégie globale» pour «insérer [le concept de développement durable] dans les programmes et les dispositifs $\gg(2003$, p. 29). Le programme de géographie de 2010 réalise cet objectif que les textes fondateurs de I'EDD ${ }^{35}$ ont officialisé.

Avec cette orientation, le programme de première peut à nouveau être considéré comme la clé de voute des études au lycée. Il est affiché comme «centré autour des questions de gestion territoriale [...] invitant les élèves à prendre conscience de la dimension prospective et stratégique de tout choix d'aménagement ». L'Europe y perd en présence thématique (un thème sur les trois années d'enseignement au lieu de charpenter le

\footnotetext{
32 Les épreuves du baccalauréat consistent depuis 1997 pour la géographie, en une épreuve de composition (première partie) et une épreuve de réalisation de croquis en réponse à un sujet (seconde partie).

33 Pour Nacima Baron, membre du groupe d'experts, le concept de territoire permet d'allier "la volonté de refondation disciplinaire (pour la construction de véritables sciences du territoire), avec de l'autre côté la tentative de renouvellement pédagogique » (Baron, 2012).

34 L'ERE vise à éduquer à travers la relation multidimensionnelle (affective, cognitive, sociale, politique, économique, esthétique) des personnes à l'environnement. L'EDD réfère explicitement au cadre de référence politique du développement durable.

${ }^{35}$ Les BOEN n० 28 du 15 juillet 2004 et $n^{\circ} 14$ du 5 avril 2007 instaurent deux phases de généralisation de I’Education en vue du Développement Durable, puis du Développement Durable.
} 
programme d'une année). L'idée d'une citoyenneté d'appartenance européenne décline au profit d'une citoyenneté d'action territoriale. La classe de terminale peut paraître en décrochage : les échelles des territoires de l'action publique ne sont guère compatibles avec les découpages en grands ensembles qui perdurent dans ce niveau de classe. La durabilité affichée comme enjeu de l'action publique en seconde et en première s'efface derrière les notions classiques de la terminale : «la puissance », «les défis du développement » et «les enjeux de la croissance ».

A cette exception près - mais elle est importante - la valeur du travail scolaire tient dans l'apprentissage de cadres de référence pour l'action en aménagement et environnement. Elle est cependant fragilisée par le fait que le programme suppose, selon nous, une expertise enseignante, non seulement pédagogique (comme en 2002), mais aussi territoriale. Or, cette seconde expertise, les savoirs spécifiques qu'elle nécessite et produit, est celle de praticiens de politiques de développement territorial, cadres de la fonction territoriale, techniciens, peut-être élus. Elle ne saurait être que la référence des pratiques enseignantes, pas ces pratiques elles-mêmes. En clair, il manque une didactique de la prospective territoriale, dont l'enseignement ne peut être déduit du seul énoncé d'une architecture conceptuelle ou d'intentions générales.

La socialisation visée par ces programmes est transformatrice : le programme est orienté vers un futur qui s'est ainsi «rapproché » de la classe. Plus exactement, le futur des territoires est un «terrain » que la classe transforme déjà en y travaillant. La limite de ce projet transformateur peut être le désarçonnement de la profession ${ }^{36}$, prise entre le défi de la référence qui lui est proposée (le décentrement disciplinaire) d'un côté et un modèle transmissif classique qu'actualise, outre le programme de terminale, la retouche des épreuves du baccalauréat qui instaure la « liste des croquis de géographie pouvant donner lieu à sujets d'examen » (Bulletin Officiel n43 du 21 novembre 2013).

\section{Assimilation à la science et récit de la mise en concurrence des territoires}

Dans le monde de la tradition qui prévaut en 2019, les programmes assimilent l'autorité dont ils sont dépositaires à « la discipline » et à « la géographie » pensées d'un bloc, tout en étant pour la première fois depuis 1995 des programmes d'« histoire-géographie ». Ils sont prolixes en contenus par lequel ils décrivent le monde en condensant un texte à développer par les professeurs.

En classe de seconde, la description permet de caractériser, distinguer les «trajectoires de développement » de pays et de régions (Inde, Brésil, Afrique australe, Arctique) mis à l'étude à travers trois thèmes (environnement, développement, mobilité). La notion floue de transition ${ }^{37}$ opère cette mise en ordre. Le programme de première fait de cet état du monde, le produit de logiques de métropolisation et de la prééminence $d^{\prime}$ « espaces productifs majeurs ». La notion de recomposition sert à repérer ces logiques responsables d'un développement différencié. En terminale, il reste à expliciter les ressorts du monde préalablement décrit, à énoncer la grandeur qui sert à mesurer les sociétés. Cette grandeur, c'est la puissance et l'influence ; sa mesure, c'est l'intégration inégale à une mondialisation qui produit « une hiérarchisation croissante des territoires à

\footnotetext{
${ }^{36}$ A partir de cette date, les nouveaux programmes sont accompagnés de la production de « ressources pour l'enseignement » accessibles en ligne à partir du site du ministère. Ces fiches de ressources n'appartiennent pas à la catégorie de la prescription. Elles en opèrent une traduction top down dans $\mathrm{I}^{\prime}$ « espace » des recommandations auxquels appartiennent aussi les formations académiques aux nouveaux programmes. Ces ressources succèdent aux publications préexistantes de commentaires des textes officiels.

37 On peut discerner quatre statuts différents de la transition: notion «clé d'analyse des grands défis contemporains », cadre de référence pour l'action (transition urbaine/villes en transition, transition environnementale), composante d'un modèle d'analyse (transition démographique) ou encore réalité comme le laisse penser ce passage du thème 4: L'Afrique australe : un espace en profonde mutation : « Les transitions, qu'elles soient démographique, économique, urbaine ou environnementale, y sont marquées par leur diversité et leur rapidité ».
} 
l'échelle mondiale». La production économique mondialisée est au centre des préoccupations ${ }^{38}$ dans un monde organisé par la mise en concurrence des territoires.

En classe de première, l'unique thème consacré à I'Union européenne prévoit cependant d'enseigner «Des politiques européennes entre compétitivité et cohésion des territoires ». La systématisation de l'étude de «la France tout au long du lycée [...] pour que les futurs citoyens aient conscience des enjeux et de leurs possibilités d'action » débouche sur des descriptions passées elles-aussi au filtre de la concurrence et de la puissance $^{39}$. Si les sociétés figurent dans le titre du thème 1 de seconde Sociétés et environnements : des équilibres fragiles, le mot même ne figure pas dans le programme de terminale. A la fin du parcours de géographie en lycée, l'économie mondialisée a « digéré » les sociétés. Sous couvert d'une autorité qui veut «faire discipline », c'est donc un récit de la concurrence mondiale des territoires qui est à faire partager.

La valeur du travail enseignant réside dans sa conformité à la prescription. Le réalisme épistémologique du programme encourage un discours enseignant de même nature. Un indice supplémentaire de cette option réaliste est l'absence pour la première fois depuis 1995 , des notions de problématique et problème qui avaient alors été introduites pour sceller le lien entre science et projet civique. La nouvelle épreuve de baccalauréat, dans sa partie intitulée « réponse à une question problématisée » demande cependant, en une formule ambigüe, que : "Le candidat [montre] qu'il a acquis des capacités d'analyse, qu'il maîtrise des connaissances, sait les sélectionner et les organiser de manière à répondre à la problématique de la question ${ }^{40}$. Cette description de plain-pied du monde (Orain, 2003) est peut-être la traduction disciplinaire de l'idée de «socle commun » (Mathiot, 2018), qui préside à la conception d'un « Nouveau baccalauréat pour construire le lycée des possibles ${ }^{41}$ (Rapport Mathiot, 2018). La liste des notions à connaître présentée à la fin de chaque niveau de classe montre la même préoccupation.

Le futur s'est éloigné de la classe de géographie entre 2010 et 2019, alors même que la préoccupation d'un continuum lycée-supérieur s'est affirmée. La dimension prospective qui « permet aux élèves [de la classe de sixième] de mieux s'approprier les dynamiques des territoires et de réfléchir aux scénarios d'avenir possibles » (BOEN, spécial n 11 du 26 novembre 2015, p. 183) n'est plus mentionnée pour le lycée. Dans les prescriptions d'enseignements communs, les apprentissages sont pensés à partir de l'auto-référence de l'exercice scolaire. La visée de socialisation s'est ainsi « scolarisée ».

\section{Conclusion}

Entre 1995 et 2019, la géographie prescrite en lycée connaît une succession de normes différentes: la rupture est une constante, l'instabilité une propriété à l'échelle de la période.

Le discours prescriptif est diversement construit : par une rhétorique du projet civique qui doit porter tous et chacun, les rédacteurs du programme comme les professeurs et bien sûr les élèves (1995) ; par une rhétorique du contrat : au programme de faire la pédagogie de la géographie pour les professeurs, à ces derniers ainsi informés de conduire les élèves jusqu'à cette géographie étude de l'organisation de l'espace (2002) ;

\footnotetext{
${ }^{38}$ D'économie, il n'est question que dans ce cadre de mondialisation. A l'inverse, il n'est pas fait mention d'économie sociale et solidaire, ni de la diversité des modèles de production et de consommation.

${ }^{39}$ Cette étude prend la forme de questions spécifiques traitant chaque thème du programme au prisme du cas français, en dehors des thèmes conclusifs de seconde et de première.

${ }^{40}$ Consultable au lien suivant:

https://www.education.gouv.fr/bo/19/Hebdo17/MENE1910707N.htm?cid_bo=141189

${ }^{41}$ A contrario, les thèmes du programme de la spécialité : histoire, géographie, géopolitique, sciences politiques, en axes et en jalons. Aucun énoncé générique ne vient préfigurer la parole de l'enseignant. Le programme présente cet enseignement comme permettant des poursuites d'études dans de nombreux cursus.
} 
par une stratégie d'ébranlement disciplinaire utilisant les cadres de référence de l'action publique et posant le futur des territoires comme terrain du travail scolaire (2010) ou en instillant un contrôle des conduites professorales et des élèves comme garantie de diffusion d'un récit fortement normalisé et pensé comme devant faire culture commune (2019).

Autrement dit, l'enjeu de reconstruction de la prescription est à chaque fois construit de façon différente. Les contenus sélectionnés servent des projets de formation changeants : fabriquer les Franco-européens de demain en les assurant d'un patrimoine et d'une culture commune (1995), sensibiliser les élèves à la complexité de la production de l'espace géographique et des problèmes d'aménagement (2002), orienter leurs préoccupations vers l'avenir des territoires en simulant des mises en action (2010), leur faire apprendre un récit partagé de la concurrence des territoires (2019).

Sensibles à l'agenda politique plus qu'aux évolutions de la géographie scientifique, les programmes scolaires en lycée opèrent des changements par à-coups mais à bas bruit. Si les filtres par lesquels passent l'appropriation scolaire de références scientifiques sont nombreux, il nous semble cependant que la recherche toujours inachevée d'une écriture des progressions d'apprentissage autre que d'autoréférence scolaire, renvoie à une difficulté de la géographie universitaire à faire référence pour cela. Faire référence, c'està-dire rendre disponible un ensemble de travaux qui aurait pour objet discuté les opérations réglant la production - dont l'écriture - scientifique de savoirs géographiques.

\section{Références bibliographiques}

Baron Yellès N., 2012, « Les tourments de la géographie scolaire face aux avancées des sciences du territoire. In : Beckouche P., Grasland C., Guérin-Pace F. \& Moisseron J.-Y. (dir.), Fonder les sciences du territoire, Paris, Karthala, p. 122-145.

Bertrand G., 1991, « Pour une didactique de la géographie physique ». In : Enseigner la géographie du collège au lycée, Amiens, CRDP Picardie, p. 15-26.

Boltanski L., Thévenot L., 1991, De la justification. Les économies de la grandeur, Paris, Gallimard.

Bonhoure G., Hagnerelle M., 2003, L'éducation relative à l'environnement et au développement durable. Rapport remis au Ministre de I'Education nationale, IGEN.

Brunet R., 1989, « Introduction au débat », L'Espace géographique, 18-2. La géographie et ses enseignements, p. 81-83.

Chevalier, J.-P., 1997, « Quatre pôles dans le champ de la géographie ? ». Cybergeo, revue européenne de géographie. URL : http://journals.openedition.org/cybergeo/6498.

Clerc P., 2002, La culture scolaire en géographie. Le monde dans la classe, Rennes, Presses universitaires de Rennes.

Davezies P., 1993, «Eléments de psychodynamique du travail », Education Permanente $n^{\circ} 116,-3$, p. $33-46$

De Cock L. (dir.), 2017, La fabrique scolaire de I'histoire, Marseille, Agone, coll. Passé \& présent.

D'Enfert R., Legris P., Verneuil Y., 2019, «La fabrique complexe et évolutive des programmes scolaires (1880-1990) ». Carrefours de l'éducation, 47, p. 23-36.

Derouet J.-L. (dir.), 2000, L'école dans plusieurs mondes, Bruxelles, de Boeck. 
Dutercq, Y., Maroy, C. (dir.). (2013). « Le développement des politiques d'accountability et leur instrumentation dans le domaine de l'éducation : une perspective francocanadienne », Éducation comparée, 11, p. 7-27.

Gaujal S., 2016, Une géographie à l'école par la pratique artistique, thèse de doctorat, géographie, Université Diderot Paris 7.

Genevois S., Leininger-Frezal, C. (dir.)2019, «L'enseignement de la géographie : entre découpages des savoirs et construction d'un curriculum de l'élémentaire à l'entrée à l'université. A partir du cas français ». Symposium, Colloque de didactiques de I'histoire, de la géographie et de l'éducation à la citoyenneté, Genève.

Kerbart-Orecchioni C., 1980, L'énonciation : de la subjectivité dans le langage, Paris, Armand Colin.

Lantheaume F., Simonian S., 2012, «La transformation de la professionnalité des enseignants : quel rôle du prescrit ? », Les Sciences de l'éducation - Pour l'Ėre nouvelle, 45-3, p. 17-38.

Lefort I., 2010, «La géographie : quelle(s) demande(s) sociale(s) pour quels publics ? » Tracés. Revue de Sciences humaines, $10 . \quad$ URL: http://journals.openedition.org/traces/4828

Legris P., 2010, L'écriture des programmes d'histoire en France (1944-2010). Sociologie historique de la production d'un instrument d'une politique éducative. Thèse de doctorat de science politique. Université Panthéon Sorbonne - Paris I.

Leininger-Frézal C., 2009, Le développement durable et ses enjeux éducatifs. Acteurs, savoirs et stratégies territoriales, thèse de doctorat de géographie, Université LumièreLyon 2.

Leininger-Frézal C., Carré C., 2016, «La géographie scolaire : d'un tournant actoriel à l'autre ? », in : Thémines J.-F., Doussot S., Acteurs et action. Perspective en didactiques de l'histoire et de la géographie, Caen, Presses universitaires de Caen, p. 227-242.

Martinand J.-L., 2011, «Pratiques de référence et problématique de la référence curriculaire », in : Terrisse A. (dir.) Didactique des disciplines, Bruxelles, de Boeck, p. 1724.

Mathiot P., 2018, Un nouveau baccalauréat pour construire le lycée des possibles. Rapport remis au Ministre de l'Education nationale.

Mons N., 2007, Les nouvelles politiques éducatives. Paris: Presses Universitaires de France.

Orain O., 2003, Le plain-pied du monde. Postures épistémologiques et pratiques d'écriture dans la géographie française au XXe siècle, thèse de doctorat de géographie, Université Panthéon-Sorbonne-Paris I.

Philippe G., 2002, « L'appareil formel de l'effacement énonciatif et la pragmatique des textes sans locuteur », in : Amossy R. (éd.). Pragmatique et analyse des textes. Tel-Aviv : Université de TelAviv), 17-34.

Prairat E. (dir.), 2009, De la déontologie enseignante, Paris, Presses Universitaires de France.

Prost A., 1998, « Un couple scolaire », Espaces Temps, n66-67, Histoire/géographie 1. L'arrangement, p. 55-64. 
Provenzano P., 2010, « Effacement énonciatif et doxa dans le discours théorique : I'exemple de Julia Kristeva », Argumentation et Analyse du Discours, 5. URL : http://journals.openedition.org/aad/973

Rabatel A., 2004, "Effacement énonciatif et effets argumentatifs indirects dans l'incipit du Mort qu'il faut de Semprun », Semen, 17. URL : http://semen.revues.org/2334

Retaillé D., 1991, «La transposition didactique du système monde », L'Information géographique, 1, p. 32-35.

Robic M.-C., Rosemberg M., 2016, Géographie aujourd'hui. Enseigner la géographie au collège et au lycée. ADAPT/SNES.

Reuter Y. (éd.), 2008, Dictionnaire des concepts fondamentaux des didactiques, Bruxelles, de Boeck.

Reuter Y., 2007, «La conscience disciplinaire ». Éducation et didactique, vol 1 - 2, p. 5571.

Thémines J.-F., 2011, Savoir et savoir enseigner le territoire, Toulouse, Presses universitaires du Mirail.

Vergnolle-Mainar C., 2011, La géographie dans l'enseignement. Une discipline en dialogue, Rennes, Presses universitaires de Rennes.

Vion R., 2001, " "Effacement énonciatif" et stratégies discursives », in : De Mattia, M. \& Joly A. (éds). De la syntaxe à la narratologie énonciative. Paris - Gap : Ophrys, p. 331354. 
Tableau $\mathrm{n}^{\circ} 1$ : Organisation des contenus des programmes de géographie des lycées généraux

\begin{tabular}{|c|c|c|c|c|}
\hline $\begin{array}{l}\text { Niveaux de } \\
\text { classe }\end{array}$ & $\begin{array}{l}\text { Programmes de } 1995 \text { (BOEN, n¹2, } \\
29 \text { juin 1995) }\end{array}$ & $\begin{array}{l}\text { Programmes de } 2002 \text { (JO, } 10 \text { juillet } \\
2002, \text { BOEN } n^{\circ} 7,3 \text { octobre 2002) }\end{array}$ & $\begin{array}{l}\text { Programmes de } 2010-2013 \text { (BOEN } \\
\text { spécial } n^{\circ} 4,29 \text { avril } 2010 ; \text { BOEN } \\
\text { spécial } n^{\circ} 9,30 \text { septembre } 2010 ; \text { BOEN } \\
n^{\circ} 42,14 \text { novembre 2013) }\end{array}$ & Programmes de 2019 \\
\hline Seconde & $\begin{array}{l}\text { LES HOMMES ET LA TERRE } \\
\text { I- La terre, planète des hommes } \\
\text { II - Les sociétés humaines face aux } \\
\text { ressources et aux contraintes de la } \\
\text { Terre } \\
\text { III - Les sociétés humaines } \\
\text { organisent et aménagent leur } \\
\text { territoire }\end{array}$ & $\begin{array}{l}\text { LES HOMMES OCCUPENT ET } \\
\text { AMENAGENT LA TERRE } \\
\text { Plus de six milliards d'hommes sur la } \\
\text { Terre } \\
\text { Nourrir les hommes } \\
\text { L'eau, entre abondance et rareté } \\
\text { Dynamiques urbaines et } \\
\text { environnement urbain } \\
\text { Les sociétés face aux risques } \\
\text { Les littoraux espaces attractifs } \\
\text { Les montagnes entre tradition et } \\
\text { nouveaux usages }\end{array}$ & $\begin{array}{l}\text { SOCIETES ET DEVELOP-PEMENT } \\
\text { DURABLE } \\
\text { Thème introductif - Les enjeux du } \\
\text { développement } \\
\text { Thème } 2 \text { - Gérer les ressources } \\
\text { terrestres } \\
\text { Thème } 3 \text { - Aménager la ville } \\
\text { Thème } 4 \text { - Gérer les espaces terrestres }\end{array}$ & $\begin{array}{l}\text { ENVIRONNEMENT, DEVELOPPEMENT, } \\
\text { MOBILITE : LES DEFIS D'UN MONDE EN } \\
\text { TRANSITION } \\
\text { Thème } 1 \text { : Sociétés et environnements : des } \\
\text { équilibres fragiles } \\
\text { Thème } 2 \text { : Territoires, populations et } \\
\text { développement : quels défis? } \\
\text { Thème } 3: \text { Des mobilités généralisées } \\
\text { Thème } 4 \text { conclusif : L'Afrique australe : un } \\
\text { espace en profonde mutation }\end{array}$ \\
\hline $\begin{array}{l}\text { Première } \\
\text { (ES et } L \text { pour } \\
2002 \text { et 2010) }\end{array}$ & $\begin{array}{l}\text { LA FRANCE EN EUROPE } \\
\text { ET DANS LE MONDE } \\
\text { I- La France en perspective } \\
\text { II - Le territoire français et son } \\
\text { organisation } \\
\text { III - Etats et régions en France et en } \\
\text { Europe }\end{array}$ & $\begin{array}{l}\text { L'EUROPE, LA FRANCE }\left(1^{\mathrm{e}} \mathrm{ES}, \mathrm{L}\right) \\
\text { Introduction : qu'est-ce que l'Europe } \\
\text { ? } \\
\text { I- L'Europe des États } \\
\text { II - Réseaux et flux en Europe et en } \\
\text { France } \\
\text { III - La France et son territoire - } \\
\text { Métropole et DOM-TOM } \\
\text { IV - Les régions en France et en } \\
\text { Europe }\end{array}$ & $\begin{array}{l}\text { FRANCE ET EUROPE : DYNAMIQUES } \\
\text { DES TERRITOIRES DANS LA } \\
\text { MONDIALISATION } \\
\text { Thème } 1 \text { - Comprendre les territoires } \\
\text { de proximité } \\
\text { Thème } 2 \text { - Aménager et développer le } \\
\text { territoire français } \\
\text { Thème } 3-\text { L'Union européenne : } \\
\text { dynamiques de développement des } \\
\text { territoires } \\
\text { Thème } 4 \text { - France et Europe dans le } \\
\text { monde }\end{array}$ & $\begin{array}{l}\text { LES DYNAMIQUES D'UN MONDE EN } \\
\text { RECOMPOSITION } \\
\text { Thème } 1: \text { La métropolisation : un processus } \\
\text { mondial différencié } \\
\text { Thème } 2: \text { Une diversification des espaces et } \\
\text { des acteurs de la production } \\
\text { Thème } 3: \text { Les espaces ruraux : } \\
\text { multifonctionnalité ou fragmentation? } \\
\text { Thème } 4 \text { conclusif : La Chine : des } \\
\text { recompositions spatiales multiples }\end{array}$ \\
\hline $\begin{array}{l}\text { Terminale } \\
\text { (ES et L pour } \\
2002 \text { et 2013) }\end{array}$ & $\begin{array}{l}\text { L'ESPACE MONDIAL } \\
\text { I - L'organisation géographique du } \\
\text { monde } \\
\text { II - Trois puissances économiques } \\
\text { mondiales } \\
\text { III - Quelques problèmes } \\
\text { géographiques mondiaux à } \\
\text { l'échelle continentale }\end{array}$ & $\begin{array}{l}\text { L'ESPACE MONDIAL } \\
\text { I- Un espace mondialisé } \\
\text { II - Les trois grandes aires de } \\
\text { puissance dans le monde } \\
\text { III - Des mondes en quête de } \\
\text { développement }\end{array}$ & $\begin{array}{l}\text { MONDIALISATION ET DYNAMIQUES } \\
\text { GEOGRAPHIQUES DES TERRITOIRES } \\
\text { Thème } 1 \text { Introductif - Clés de lectures } \\
\text { d'un monde complexe } \\
\text { Thème } 2 \text { - Les dynamiques de la } \\
\text { mondialisation } \\
\text { Thème } 3 \text { - Dynamiques géographiques } \\
\text { de grandes aires continentales }\end{array}$ & $\begin{array}{l}\text { LES TERRITOIRES DANS LA MONDIALISATION : } \\
\text { ENTRE INTEGRATIONS ET RIVALITES } \\
\text { Thème } 1-\text { Mers et océans : au cœur de la } \\
\text { mondialisation } \\
\text { Thème } 2-\text { Dynamiques territoriales, } \\
\text { coopérations et tensions dans la } \\
\text { mondialisation } \\
\text { Thème } 3-\text { L'Union européenne dans la } \\
\text { mondialisation : des dynamiques complexes } \\
\text { Thème conclusif }- \text { La France et ses régions }\end{array}$ \\
\hline
\end{tabular}




\begin{tabular}{|l|l|l|l|l|}
\hline & & & $\begin{array}{l}\text { dans l'Union européenne et dans la } \\
\text { mondialisation } \\
\text { recompositions }\end{array}$ & \\
lignes de force & et
\end{tabular}

Tableau $^{\circ} 2$ : Relevés de résultats de l'analyse de discours mise en œuvre sur les programmes de géographie des lycées généraux

\begin{tabular}{|c|c|c|c|c|}
\hline & Programmes de 1995 & Programmes de 2002 & Programmes de 2010-2013 & Programmes de 2019 \\
\hline \multicolumn{5}{|c|}{ Objets construits par effacement énonciatif } \\
\hline Objets construits & $\begin{array}{l}\text { L'enseignement de l'histoire et de } \\
\text { la géographie } \\
\text { Le projet éducatif de } \\
\text { l'enseignement } \\
\text { Les programmes }\end{array}$ & $\begin{array}{l}\text { Les programmes } \\
\text { Les contenus }\end{array}$ & $\begin{array}{l}\text { Le programme } \\
\text { Les objectifs d'apprentissage } \\
\text { Le développement durable (en } \\
\text { seconde) }\end{array}$ & $\begin{array}{l}\text { Les disciplines } \\
\text { L'enseignement } \\
\text { La géographie }\end{array}$ \\
\hline $\begin{array}{l}\text { Procédés de dynamisation de } \\
\text { ces objets (par mise en } \\
\text { œuvre d'énoncés } \\
\text { objectivants) }\end{array}$ & $\begin{array}{l}\text { Enoncés doxiques et axiologiques } \\
\text { décrivant les propriétés générales } \\
\text { des objets construits. } \\
\text { Par exemple: } \\
\text { L'enseignement de l'histoire et de } \\
\text { la géographie, délibérément ouvert } \\
\text { sur le temps présent ne dissocie pas } \\
\text { transmission d'un héritage et d'une } \\
\text { culture, formation intellectuelle et } \\
\text { éducation à la citoyenneté. } \\
\text { Les programmes d'histoire et de } \\
\text { géographie du lycée doivent [...] } \\
\text { répondre à des finalités } \\
\text { (connaissances, apprentissage de la } \\
\text { réflexion, exercice de la raison } \\
\text { critique) }\end{array}$ & $\begin{array}{l}\text { Enoncés doxiques et axiologiques } \\
\text { Par exemple: } \\
\text { Les programmes d'histoire- } \\
\text { géographie [...] leur donnent une } \\
\text { vision dynamique et distanciée du } \\
\text { monde, fondement nécessaire } \\
\text { d'une citoyenneté qui devient au } \\
\text { lycée une réalité effective. } \\
\text { Chaque thème s'articule autour } \\
\text { d'une ou deux études de cas qui } \\
\text { mettent en place les } \\
\text { problématiques nécessaires à } \\
\text { l'appropriation des savoirs et } \\
\text { constituent l'apprentissage du } \\
\text { raisonnement géographique. }\end{array}$ & $\begin{array}{l}\text { Enoncés doxiques et axiologiques } \\
\text { Par exemple: } \\
\text { Le programme de seconde est } \\
\text { centré sur les questions de } \\
\text { développement durable } \\
\text { En croisant les dimensions sociales, } \\
\text { économiques et } \\
\text { environnementales, on s'interroge } \\
\text { sur la façon dont les sociétés } \\
\text { humaines améliorent leurs } \\
\text { conditions de vie [...] Le } \\
\text { développement durable apparaît } \\
\text { ainsi comme une autrefaçon de lire } \\
\text { le monde, de le penser et de le } \\
\text { gérer. } \\
\text { Mise en tableau des objectifs } \\
\text { d'apprentissage }\end{array}$ & $\begin{array}{l}\text { Enoncés doxiques } \\
\text { Par exemple: } \\
\text { Des disciplines pour comprendre et } \\
\text { agir - Par l'étude du passé et } \\
\text { l'examen du présent, l'histoire et la } \\
\text { géographie enseignées au lycée } \\
\text { transmettent aux élèves des } \\
\text { connaissances précises et diverses sur } \\
\text { un large empan historique, } \\
\text { s'étendant de l'Antiquité à nos jours. } \\
\text { L'enseignement d'histoire- } \\
\text { géographie développe des } \\
\text { connaissances et construit des } \\
\text { capacités et méthodes spécifiques }\end{array}$ \\
\hline \multicolumn{5}{|c|}{ Constructions discursives de la référence géographique, du professeur et de l'élève } \\
\hline $\begin{array}{l}\text { Construction de l'image de la } \\
\text { référence }\end{array}$ & $\begin{array}{l}\text { Introduite par l'objet: projet } \\
\text { éducatif de l'enseignement de } \\
\text { l'histoire et de la géographie } \\
\text { Le projet éducatif de } \\
\text { l'enseignement de l'histoire et de la } \\
\text { géographie repose sur une étude }\end{array}$ & $\begin{array}{l}\text { Rattachée à l'objet programme } \\
\text { L'intitulé du programme renvoie } \\
\text { explicitement à l'objet fondamental } \\
\text { de la géographie qui étudie le } \\
\text { monde en tant que territoire de } \\
\text { l'humanité. II permet donc }\end{array}$ & $\begin{array}{l}\text { Rattachée à l'objet développement } \\
\text { durable en seconde } \\
\text { Par ses concepts, ses outils et ses } \\
\text { méthodes conduisant à une mise en } \\
\text { relation permanente des sociétés } \\
\text { humaines avec leurs }\end{array}$ & $\begin{array}{l}\text { Un objet de discours: la géographie, } \\
\text { Introduit lui-même par l'objet } \\
\text { enseignement } \\
\text { Finalités - La géographie vise à } \\
\text { comprendre comment les individus et } \\
\text { les sociétés organisent leur espace, }\end{array}$ \\
\hline
\end{tabular}




\begin{tabular}{|c|c|c|c|c|}
\hline & $\begin{array}{l}\text { précise des contenus scientifiques } \\
\text { de ces disciplines : vocabulaire et } \\
\text { notions essentielles, nouveaux } \\
\text { problèmes et nouvelles approches, } \\
\text { langages spécifiques. II se nourrit } \\
\text { des problématiques et des } \\
\text { avancées de la recherche } \\
\text { universitaire }\end{array}$ & $\begin{array}{l}\text { d'aborder la problématique de } \\
\text { l'organisation de l'espace par les } \\
\text { hommes }\end{array}$ & $\begin{array}{l}\text { environnements, la géographie } \\
\text { apporte une contribution majeure } \\
\text { aux approches du développement } \\
\text { durable, réactivant les questions de } \\
\text { développement et les replaçant au } \\
\text { cœur des débats de société }\end{array}$ & s'y développent, le transforment. \\
\hline $\begin{array}{l}\text { Construction de l'image du } \\
\text { professeur }\end{array}$ & $\begin{array}{l}\text { Introduite par les objectifs } \\
\text { généraux qui est le premier attribut } \\
\text { de l'objet programmes } \\
\text { Une ancienne tradition laisse aux } \\
\text { enseignants une grande liberté } \\
\text { d'approche pédagogique. Ils } \\
\text { peuvent, pourvu que les objectifs et } \\
\text { la cohérence d'ensemble soient } \\
\text { respectés, choisir leur itinéraire } \\
\text { annuel et insister plus ou moins sur } \\
\text { tel ou tel thème. Cette liberté } \\
\text { permet à chaque professeur } \\
\text { d'adopter le style d'enseignement } \\
\text { qu'il pense le plus efficace et } \\
\text { d'adapter ses méthodes aux élèves } \\
\text { et à leurs besoins. }\end{array}$ & $\begin{array}{l}\text { Introduite à partir des contenus } \\
\text { Afin que ces finalités affichées } \\
\text { soient réalisables, les contenus des } \\
\text { programmes sont nettement } \\
\text { délimités tout en préservant la } \\
\text { liberté pédagogique des } \\
\text { enseignants. } \\
\text { Ces thèmes sont traités de façon } \\
\text { problématisée au travers } \\
\text { d'exemples choisis sur tous les } \\
\text { continents et abordés aux } \\
\text { différentes échelles. }\end{array}$ & $\begin{array}{l}\text { Construite en fonction des objectifs } \\
\text { d'apprentissage } \\
\text { Dans la construction de son } \\
\text { enseignement, en s'appuyant sur } \\
\text { les acquis du socle commun de } \\
\text { connaissances et de compétences, } \\
\text { le professeur vise comme objectifs } \\
\text { d'apprentissage les connaissances, } \\
\text { les capacités et les méthodes } \\
\text { définies dans les programmes }\end{array}$ & $\begin{array}{l}\text { Introduite par l'enseignement } \\
\text { l'enseignement associe des temps } \\
\text { dédiés : - a la transmission des } \\
\text { connaissances par les professeurs et } \\
\text { d'écoute active de la part des élèves; } \\
\text { - à l'étude de sources, à l'analyse } \\
\text { approfondie et critique de documents } \\
\text { variés (cartes, textes, iconographie, } \\
\text { vidéos...) et à la réalisation de } \\
\text { croquis. }\end{array}$ \\
\hline $\begin{array}{l}\text { Construction de l'image de } \\
\text { l'élève }\end{array}$ & $\begin{array}{l}\text { Attachée à l'objet enseignement de } \\
\text { l'histoire et de la géographie: } \\
\text { A la sortie du lycée, les élèves } \\
\text { doivent conserver de } \\
\text { l'enseignement de l'histoire et de la } \\
\text { géographie une vision positive avec } \\
\text { le sentiment que ces disciplines, } \\
\text { constamment ouvertes sur les idées } \\
\text { et les techniques d'aujourd'hui, ont } \\
\text { forgé aussi bien leur sens critique } \\
\text { que leur sens civique fondés sur } \\
\text { l'appropriation d'un patrimoine et } \\
\text { d'une culture. }\end{array}$ & $\begin{array}{l}\text { Construite en relation avec l'étude } \\
\text { de cas, elle-même fortement } \\
\text { attachée aux contenus: } \\
\text { Les études de cas, toujours } \\
\text { resituées dans un cadre plus large, } \\
\text { visent à fournir aux élèves les } \\
\text { moyens de comprendre le monde } \\
\text { dans lequel ils vivent }\end{array}$ & $\begin{array}{l}\text { Attachée en seconde à l'objet } \\
\text { développement durable, sans } \\
\text { nommer les élèves, mais en ciblant } \\
\text { un objectif général: } \\
\text { Cette démarche fournit l'occasion } \\
\text { de progresser dans la maîtrise des } \\
\text { approches systémiques et dans la } \\
\text { prise en compte de la complexité et } \\
\text { de la hiérarchie des facteurs } \\
\text { d'explication }\end{array}$ & 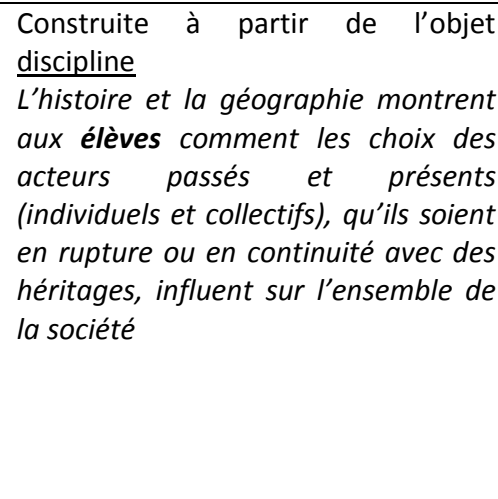 \\
\hline
\end{tabular}




\section{Résumé}

Les programmes de géographie du lycée parus entre 1995 et 2019 en France sont analysés en tant que norme institutionnelle fixant tout à la fois les contenus de géographie sélectionnés et le travail attendu des professeurs et des élèves. Ils engagent aussi un rapport spécifique avec la géographie scientifique et avec le groupe des enseignants. Ils expriment enfin la visée officielle de formation à laquelle la discipline doit contribuer pour chaque nouvelle génération. L'analyse de discours montre que des normes différentes se succèdent pendant la période et que la formation initiale en géographie voulue par l'institution prend des orientations parfois antagoniques.

\section{Mots-clés :}

didactique, géographie scolaire, norme institutionnelle, programmes scolaires

\section{Abstract}

The high school geography curriculum published between 1995 and 2019 in France are analysed as an official standard that prescribes the organization of geographic content and the expected work of teachers and students. This standard defines the relationship that school contents must have with scientific geography and that the educational institution must have with the professional group of teachers. It expresses the formal objective of the expected training in geography for each new generation. The analysis of the discourse shows that different standards follow one another, that the scientific reference is used in a very variable way and that the formal training required for the young generations sometimes takes opposite directions.

\section{Keywords}

Didactics, high school geography, official standard, formal curriculum 